\title{
Smartphone application for celiac patients: assessing its effect on gastrointestinal symptoms in a randomized controlled clinical trial
}

\section{Zeinab Nikniaz}

Liver and gastrointestinal diseases research center, Tabriz University of medical sciences, Tabriz, Iran

Masood Shirmohammadi

Liver and gastrointestinal diseases research center, Tabriz University of medical sciences, Tabriz, Iran

Elham Masserat

Department of Health Information Technology, School of Health Management and Medical Informatics,

Tabriz University of Medical Sciences, Tabriz, Iran

Zahra Akbari Namvar ( $\square$ zahranamvar1993@gmail.com )

\section{Research article}

Keywords: celiac disease; gastrointestinal symptoms; smartphone application; GSRS score

Posted Date: June 30th, 2020

DOI: https://doi.org/10.21203/rs.3.rs-33137/v1

License: (1) This work is licensed under a Creative Commons Attribution 4.0 International License.

Read Full License 


\section{Abstract}

Background: We aimed to study the effectiveness of a "celiac" smartphone application on gastrointestinal symptoms in celiac patients.

Methods: In the present randomized controlled clinical trial, 60 celiac patients were randomly assigned to receive education through a smartphone application $(n=30)$ or conventional clinical education $(n=30)$. We assessed the gastrointestinal symptoms using the gastrointestinal symptom rating scale (GSRS) questionnaire at baseline and three months after interventions. The GSRS total score, celiac disease GSRS (CD-GSRS) score, abdominal pain, reflux, diarrhea, constipation, and indigestion scores were calculated.

Results: Out of 60 randomized patients, 58 patients completed the study. In comparison to baseline, the mean score of GSRS total score $(p=0.04)$, CD-GSRS score $(p=0.001)$, and indigestion subscore $(p<0.001)$ were significantly decreased in the intervention group. In the control group, compared to baseline, the mean scores of CD-GSRS score $(p=0.03)$ and abdominal pain subscore $(p=0.04)$ were significantly reduced; and the mean score of reflux $(p=0.01)$ subscore was significantly increased. The results of the between-group comparisons showed that although the mean GSRS and CD-GSRS total scores were lower in the intervention group compared with the control group, the differences were not statistically significant. In terms of GSRS subscores, there was a significant difference between the two groups only in the mean score of indigestion $(P=0.01)$.

Conclusion: According to the results, smartphone application had a significantly positive effect on indigestion symptoms compared with routine clinic education. However, more large studies with longer duration of the follow-up period are needed to assure this approach

Trial registration: The protocol of the study was registered in the Iranian registry of clinical trials (IRCT code: IRCT20170117032004N2). trial registry webpage: : https://www.irct.ir/user/trial/40187/view. Trial registry date: 2019.6 .26

\section{Background}

Celiac disease (CD) is an autoimmune entrities characterized by gluten intolerance ${ }^{1}$. It is associated with the destruction of small intestine mucosa and accompanied by different gastrointestinal (GI) and non-GI symptoms ${ }^{1}$. Avoiding life-long gluten-containing foods is the only available treatment for these patients 2. However, due to different reasons such as lack of knowledge, inadequate labeling and high cost of gluten-free products, etc., strict compliance to a gluten-free diet (GFD) is challenging ${ }^{3}$. In Iran, vouchers are given to patients to buy essential gluten-free products such as bread and floor. However, lack of knowledge about gluten-containing products is one of the main problems. Although individualized education by an expert dietitian is considered the standard of care, it is costly and time-consuming. So, different methods were studied to overcome these limitations. In this regard, various online education 
programs have been designed and applied in the adult ${ }^{4}$ and children celiac patients ${ }^{5}$ and their positive effect has been reported. However, this method needs internet access. In another study, the positive effects of the text-message intervention were reported ${ }^{6}$. However, a limited amount of data can be transferred through this method. Recently, due to the global increase in the use of smartphones, transferring health messages through smartphone applications has grown. This method was used for promoting health-related behaviors ${ }^{7-11}$ and in term of diet therapy, the positive effect of a smartphone application in weight reduction programs have been shown ${ }^{12-14}$. Previously, an English language smartphone application, "MyHealthyGut", was developed for celiac patients ${ }^{15}$ and its positive effect on gastrointestinal symptoms had been reported ${ }^{16}$. However, concerning the differences in the list of glutenfree products between countries, a specific application should be developed for each culture and country. There is not an inclusive Persian application for celiac patients that covers all aspects of the GFD. Thus, in the present study, we aimed to study the effectiveness of a smartphone application on GI symptoms in celiac patients.

\section{Methods}

\section{Participants:}

In the present two-arm, parallel design randomized controlled clinical trial, the patients were selected randomly from the East-Azerbaijan celiac disease registry database, based on the following criteria: age>18 years, being at least 6 months post-diagnosis, and use a smartphone. The patients who had other chronic inflammatory GI diseases, diabetes, or autoimmune, psychiatric, or neurological disease that could interfere with assessments were excluded. All patients were diagnosed by compatible duodenal histological findings, serology markers, and clinical symptoms.

In the present study, 65 celiac patients were selected out of 80 patients who had eligibility criteria. Five patients did not consent to participate in the study and 60 patients were randomly assigned into two groups using the GraphPad randomization software tool. The randomization process was conducted by a third person not involved in the research protocol. All patients have received GFD recommendations in the celiac clinic of Imam Reza hospital with the help of leaflets. The patients in the intervention group $(n=30)$ received the celiac application in addition to routine education in the clinic. The patients in the control group $(n=30)$ received only weekly routine education in the clinic with the help of leaflets. Both programs had the same content. The outcome assessor and statistition were blind to group allocation.

The "celiac" is a Persian language application that was designed by our research team and confirmed in a team of 10 nutritionists and gastroenterologists. "celiac application" was designed for providing different information regarding CD and GFD in seven sections including: "About Disease", "Gluten-free Diet”, "Gluten-free Drugs and Supplements", "Gluten-free Food Labeling”, “Gluten-free Cooking”, "Calculations" and "Celiac Centers in Iran". In the "About Disease" section various information such as disease causes, symptoms, diagnosis, and treatments were provided. In the "Gluten-free Diet" section different foods were marked by three colors: green for allowed foods, red for prohibited foods, and orange 
for suspicious foods. The generic name and also factory name of the gluten-free drug's list which are introduced by Iran's Food and Drugs Organization was provided in the "Gluten-free Drugs and

Supplements" section. In the "Gluten-free Food Labeling", the name of different food ingredients that may contain gluten were provided. In the "Gluten-free Cooking" the cooking methods of different foods with gluten-free ingredients were provided. The calculation section allowed patients to calculate their body mass index (BMI) and also to register their tissue transglutaminase antibodies for follow up. Finally, the "Celiac Disease Centers" section listed the address of CD clinics and gluten-free food providers in different provinces of Iran for more access of patients to gluten-free foods when they are on the journey. The patients were asked to use it for getting the required information for three months.

\section{Outcome measure:}

The difference in the severity of gastrointestinal symptoms between the two groups was the outcome of interest that was assessed by the Gastrointestinal Symptom Rating Scale score (GSRS) questionnaire. This questionnaire is a seven-point Likert scale with 15 questions that has response options ranging from "no problem (0)" to "severe discomfort (6)". The questionnaire is divided into 5 domains that cover the gastrointestinal system: diarrhea (3 questions; score range: $0-18$ ), constipation (3 questions; score range: $0-18$ ), abdominal pain (3 question; score range: 0-18), reflux (2 questions; score range: 0-12), and indigestion (4 questions; score range: 0-24). The questionnaire was translated into the Persian language and validated in our population previously ${ }^{17}$. The questionnaire was also modified to fit patients suffering from celiac disease (CD-GSRS). CD-GSRS contains 10 questions from the GSRS and includes the following domains: diarrhea (3 questions; score range: 0-18), abdominal pain (3 questions; score range: $0-18$ ), and indigestion (4 questions; score range: $0-24)^{18}$. Higher scores represent worse symptoms.

This study adheres to CONSORT guidelines.

\section{Statistical analysis:}

Originally, the study was designed and powered to detect the effects of a smartphone application on patients' knowledge and adherence level which were taken as the primary endpoints (Data were presented in our previous publication). In this study, we presented the secondary outcome variables, the gastrointestinal effects of our intervention. The sample size was calculated assuming an effect size of 0.4 , the significance level of 0.05 and power of $80 \%$, the minimum sample size needed to detect a significant between-group difference in adherence level was 21 participants in each group (total sample: 52). Considering the probability of $40 \%$ attrition rate, the desired sample size was 30 patients in each group.

SPSS 21.0 was used for statistical analysis. Skewness and Kurtosis were used for testing the assumption of normality. The quantitative values were reported as mean and standard deviation, and the qualitative variables were reported in frequency and percentage. The with-in group comparisons were done by Paired t-test and the between-group changes were analyzed by independent sample t-test and 
one-way analysis of covariance (ANCOVA) with adjusting for age, sex, disease duration, educational level, and baseline values. For comparing the nominal and ordinal variables, the chi-square and Mann Whitney$\mathrm{U}$ tests were used.

\section{Results}

In the present study out of 60 patients who were randomly divided into two groups, the questionnaire of one patient in the intervention group was incomplete and one patient in the control group lost to follow up (Figure 1). The data were analyzed on 29 patients in each group. Table 1 presents the baseline characteristics of patients. As indicated, the baseline characteristics were not significantly different between groups $(P>0.05)$.

Table 1: The baseline characteristics of participants stratified by groups

\begin{tabular}{|c|c|c|c|}
\hline Variable & Intervention $(\mathrm{n}=29)$ & Control $(n=29)$ & p-value \\
\hline Age $($ Mean \pm SD) Years & $36.04 \pm 8.54$ & $38.62 \pm 9.88$ & $0.28^{*}$ \\
\hline Sex n (\%) male/female & $8(27.5) / 21(72.4)$ & $10(34.4) / 19(65.5)$ & $0.22 * *$ \\
\hline \multicolumn{4}{|l|}{ Education n (\%) } \\
\hline$\leq$ Diploma & $15(51.72)$ & $20(68.9)$ & \multirow[t]{2}{*}{$0.18^{* *}$} \\
\hline College education & $14(48.27)$ & $9(31.0)$ & \\
\hline Marital status & & & 0.32 \\
\hline Single & $8(27.58)$ & $7(24.1)$ & \\
\hline Married & $21(72.4)$ & $22(75.8)$ & \\
\hline Positive Family history & $2(6.8)$ & $1(3.4)$ & 0.38 \\
\hline Marsh & & & 0.35 \\
\hline I & $4(13.79)$ & $3(10.34)$ & \\
\hline II & $6(20.68)$ & $5(17.24)$ & \\
\hline IIIa & $6(20.68)$ & $9(31.03)$ & \\
\hline IIIlb & $8(27.58)$ & $7(24.13)$ & \\
\hline IIIc & $5(17.24)$ & $5(17.24)$ & \\
\hline Presence of comorbidities & $6(20.68)$ & $9(31.0)$ & 0.55 \\
\hline Disease duration & $5.32 \pm 4.05$ & $3.51 \pm 2.26$ & 0.16 \\
\hline Occupation & & & 0.62 \\
\hline Employed & $10(34.4)$ & $11(37.9)$ & \\
\hline Student & $3(10.3)$ & $3(10.3)$ & \\
\hline House wife & $16(55.1)$ & 15 (51.7) & \\
\hline
\end{tabular}

BMI: Body mass index; SD: standard deviation *P-value of independent t-test

** $\mathrm{P}$-value of chi-square

The mean changes from baseline of GSRS scores and subscores stratified by groups are shown in Figure 2. As depicted, in comparison to baseline, the mean score of GSRS total score $(p=0.04), C D-G S R S$ score $(p=0.001)$, and indigestion subscore $(p<0.001)$ were significantly decreased in the intervention group. In the control group, compared to baseline, the mean score of CD-GSRS total score $(p=0.03)$ and abdominal pain subscore $(p=0.04)$ was significantly reduced; and the mean score of reflux $(p=0.01)$ subscore was significantly increased. 
Table 2 shows the comparison of the mean GSRS total score and subscores between the two groups. There were no significant differences between the two groups at baseline. Three months after the intervention, the mean GSRS, and CD-GSRS total scores were lower in the intervention group compared with the control group, however, the differences were not statistically significant. In terms of subscores, there was a significant difference between the two groups only in the mean score of indigestion $(P=0.01)$.

\section{Table 2: The comparison of the GSRS total scores and subscores between studied groups.}

\begin{tabular}{l|llllll} 
& \multicolumn{7}{l}{ Application group $(\mathrm{n}=29)$} & Placebo $(\mathrm{n}=29)$ & p-value* & p-value** \\
\cline { 6 - 7 } \multicolumn{1}{c}{ Variables } & Before & After & Before & After & & \\
\hline GSRS total score & $33.64 \pm 18.44$ & $27.28 \pm 15.69$ & $36.93 \pm 23.47$ & $35.09 \pm 19.05$ & 0.57 & 0.36 \\
CD-GSRS & $25.32 \pm 13.62$ & $17.48 \pm 11.13$ & $25.31 \pm 16.92$ & $22.09 \pm 13.21$ & 0.99 & \\
Abdominal pain & $6.04 \pm 4.76$ & $5.04 \pm 5.64$ & $7.80 \pm 6.01$ & $5.65 \pm 4.35$ & 0.24 & 0.31 \\
Reflux & $3.04 \pm 3.22$ & $3.75 \pm 4.02$ & $3.93 \pm 4.05$ & $6.06 \pm 4.22$ & 0.38 & 0.01 \\
Indigestion & $13.84 \pm 5.96$ & $8.20 \pm 5.3$ & $13.23 \pm 7.89$ & $12.36 \pm 7.91$ & 0.40 & 0.87 \\
Diarrhea & $5.91 \pm 7.16$ & $4.62 \pm 6.10$ & $6.96 \pm 6.75$ & $4.89 \pm 6.24$ & 0.58 & 0.40 \\
Constipation & $5.62 \pm 4.79$ & $6.45 \pm 6.97$ & $8.46 \pm 6.30$ & $7.82 \pm 7.92$ & 0.07 & 0.47 \\
\hline
\end{tabular}

GSRS: Gastrointestinal Symptom Rating Scale; CD-GSRS: celiac disease-gastrointestinal Symptom Rating Scale;

*p-value of independent t-test

**p-value of one-way ANCOVA with adjusting to age, sex, disease duration, and baseline values

\section{Discussion}

Increasing the knowledge of celiac patients about gluten-free food, drugs, and commercial products were considered as an important way of increasing their adherence to GFD and alleviating the disease symptoms ${ }^{19}$. In the present study, to overcome the limitations of the previous methods, we designed a smartphone application and assessed its efficacy on decreasing the GI symptoms in celiac patients. We showed that a smartphone application was significantly effective in decreasing indigestion in comparison to routine clinic visits. These results were consistent with the findings of a previous study that used the "MyHealthyGut" smartphone application in celiac disease. Dowd et al showed that onemonth use of application had a significantly more positive effect on GI symptoms in celiac patients ${ }^{16}$. These findings may be due to the specific characteristics of smartphone applications that increase the patient's adherence to the diet. A large amount of information can be transferred through applications ${ }^{20}$ and considering the mobility feature of them, by just installing the applications, they can be used whenever somebody needs it ${ }^{21}$. In addition to the gluten content of foods, other information such as gluten-related labeling and gluten-free drug list, etc were also provided to patients through applications. 
Previously lack of knowledge about the gluten content of commercial foods and drugs was reported as a barrier of GFD adherence ${ }^{19}$. However, using this application, this information can easily be used during shopping and dining outside the home and could increase the patient's adherence to the diet.

Although GSRS total score and some subscores (reflux, abdominal pain, diarrhea, and constipation) were lower in the intervention group compared with the control group, we did not reach a significant level. This observation may be related to the short duration of follow up and low sample size of the study.

According to the results, we showed that compared with baseline, the gastrointestinal symptoms were alleviated in the control group. This finding may be because of providing the same information in weekly visits to the clinic. So, we expect that the GI symptoms also relieve in this group.

We acknowledge the limitations of the present study such as short duration of the follow-up and low sample size. Moreover, assessing the GI symptoms was the secondary endpoint of this clinical trial, and the power calculation was not done for this aim. Also, we only included the patients who had the ability of reading and had a smartphone that limits its generalizability to all celiac patients.

\section{Conclusions}

In conclusion, a smartphone application was significantly more effective than routine clinic education in relieving indigestion. However, we did not reach a significant level in terms of other symptoms. Considering the limitations of the study, further investigations with longer follow-up duration are needed. From the practical point of view, smartphone applications can be designed according to each country's particular circumstances and can be suggested by nutritionists and physicians to use by celiac patients.

\section{List Of Abbreviations}

CD: Celiac disease; GFD: Gluten-free diet; GSRS: gastrointestinal symptom rating scale; CD-GSRS: celiac disease-gastrointestinal symptom rating scale (GSRS); ANCOVA: one-way analysis of covariance

\section{Declarations}

Ethics approval and consent to participate: The present study was approved by the Ethics Committee of Tabriz University of medical sciences (IR.TBZMED.REC.1397.697). Written informed consent was obtained from all participants.

Consent to publish: Not applicable

Availability of data and materials: The datasets supporting the conclusions of this research are included within the article.

Competing interests: Authors declare no conflict of interest. 
Funding: This project was supported by a grant from Liver and gastrointestinal diseases research center, Tabriz University of medical sciences under the grant number 60859 to develop an application and conduct the study.

Authors' contributions: ZN: Conceptualization; Funding acquisition, Supervision, Methodology, Writingreview \& editing, Formal analysis; ZAN: Project administration, Software, Writing - review \& editing; MSh: Project administration, Writing - review \& editing.

All authors have read and approved the manuscript.

Acknowledgements: The author wish to thank East Azerbaijan celiac patients for participating in the study.

\section{References}

1. Makovicky P, Makovicky P, Caja F, et al. Celiac disease and gluten-free diet: past, present, and future. Gastroenterol Hepatol Bed Bench 2020; 13: 1.

2. Bascunan KA, Vespa MC and Araya M. Celiac disease: understanding the gluten-free diet. Eur J Nutr 2017; 56: 449-459.

3. Hall N, Rubin $\mathrm{G}$ and Charnock A. Systematic review: adherence to a gluten-free diet in adult patients with coeliac disease. Aliment Pharmacol Ther 2009; 30: 315-330.

4. Sainsbury K. Online program to help people with coeliac disease manage the gluten free diet. Australian Coeliac, The 2014: 20.

5. Connan V, Marcon MA, Mahmud FH, et al. Online education for gluten-free diet teaching: Development and usability testing of an e-learning module for children with concurrent celiac disease and type 1 diabetes. Pediatr Diabetes 2019; 20: 293-303.

6. Haas K, Martin A and Park K. Text message intervention (TEACH) improves quality of life and patient activation in celiac disease: a randomized clinical trial. J Pediatr 2017; 185: 62-67. e62.

7. Díaz-Hellín P, Fontecha J, Hervás R, et al. NFC as a Childhood Obesity Treatment Tool. J Med Syst 2015; 39: 96.

8. El-Gayar O, Timsina P, Nawar N, et al. Mobile applications for diabetes self-management: status and potential. J Diabetes Sci Techno/ 2013; 7: 247-262.

9. Semper H, Povey R and Clark-Carter D. A systematic review of the effectiveness of smartphone applications that encourage dietary self-regulatory strategies for weight loss in overweight and obese adults. Obes Rev 2016; 17: 895-906.

10. Cook KA, Modena BD and Simon RA. Improvement in asthma control using a minimally burdensome and proactive smartphone application. The Journal of Allergy and Clinical Immunology: In Practice 2016; 4: 730-737. e731. 
11. Licskai CJ, Sands TW and Ferrone M. Development and pilot testing of a mobile health solution for asthma self-management: asthma action plan smartphone application pilot study. Can Respir J 2013; 20: 301-306.

12. Carter MC, Burley VJ, Nykjaer C, et al. Adherence to a smartphone application for weight loss compared to website and paper diary: pilot randomized controlled trial. J Med Internet Res 2013; 15: e32.

13. Gilliland J, Sadler R, Clark A, et al. Using a smartphone application to promote healthy dietary behaviours and local food consumption. BioMed Res Int 2015; 2015.

14. Laing BY, Mangione $\mathrm{CM}$, Tseng $\mathrm{C}-\mathrm{H}$, et al. Effectiveness of a smartphone application for weight loss compared with usual care in overweight primary care patients: a randomized, controlled trial. Ann Intern Med 2014; 161: S5-S12.

15. Dowd AJ, Jackson C, Tang KTY, et al. MyHealthyGut: development of a theory-based self-regulatory app to effectively manage celiac disease. Mhealth 2018; 4: 19. 2018/07/28. DOI: 10.21037/mhealth.2018.05.05.

16. Dowd AJ, Warbeck CB, Tang KT, et al. MyHealthyGut: Findings from a pilot randomized controlled trial on adherence to a gluten-free diet and quality of life among adults with celiac disease or gluten intolerance. Digit Health 2020; 6: 2055207620903627. 2020/02/20. DOI: $10.1177 / 2055207620903627$.

17. Mazaheri M and Khoshouei MS. Comparison between Psychometric Characteristics of Persian Version of the Gastrointestinal Symptoms Rating Scale in Functional Gastrointestinal Disorders and Normal Groups. Govaresh 2012; 17: 17-24.

18. Leffler DA, Kelly CP, Green PH, et al. Larazotide acetate for persistent symptoms of celiac disease despite a gluten-free diet: a randomized controlled trial. Gastroenterology 2015; 148: 1311-1319. e1316.

19. Rajpoot $P$, Sharma A, Harikrishnan S, et al. Adherence to gluten-free diet and barriers to adherence in patients with celiac disease. Indian J Gastroentero/ 2015; 34: 380-386.

20. Coughlin SS, Whitehead M, Sheats JQ, et al. Smartphone applications for promoting healthy diet and nutrition: a literature review. Jacobs journal of food and nutrition 2015; 2: 021.

21. Free C, Phillips G, Galli L, et al. The effectiveness of mobile-health technology-based health behaviour change or disease management interventions for health care consumers: a systematic review. PLoS Med 2013; 10: e1001362.

\section{Figures}




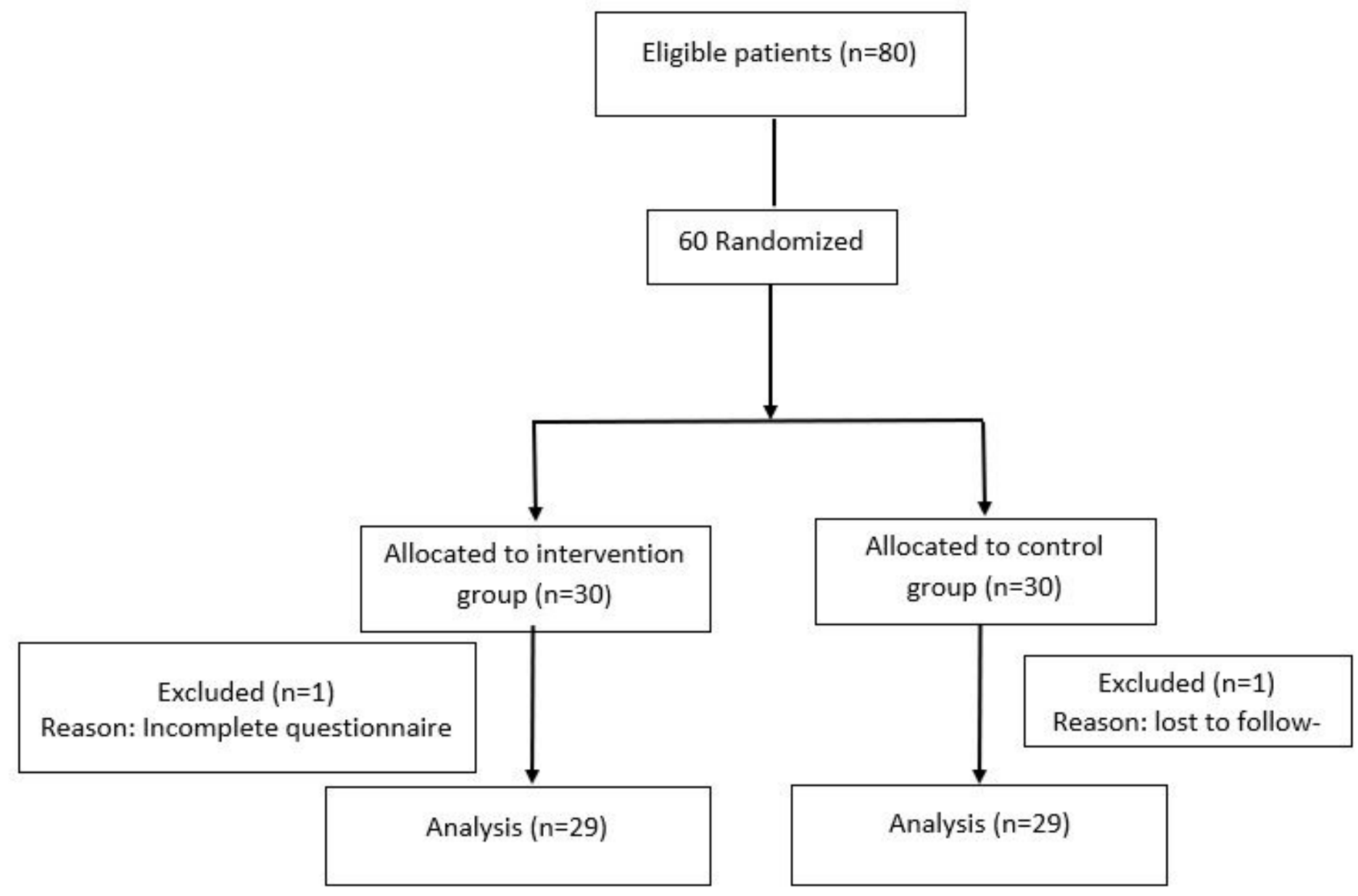

Figure 1

Flow chart for patient enrolment, randomization, and retention 


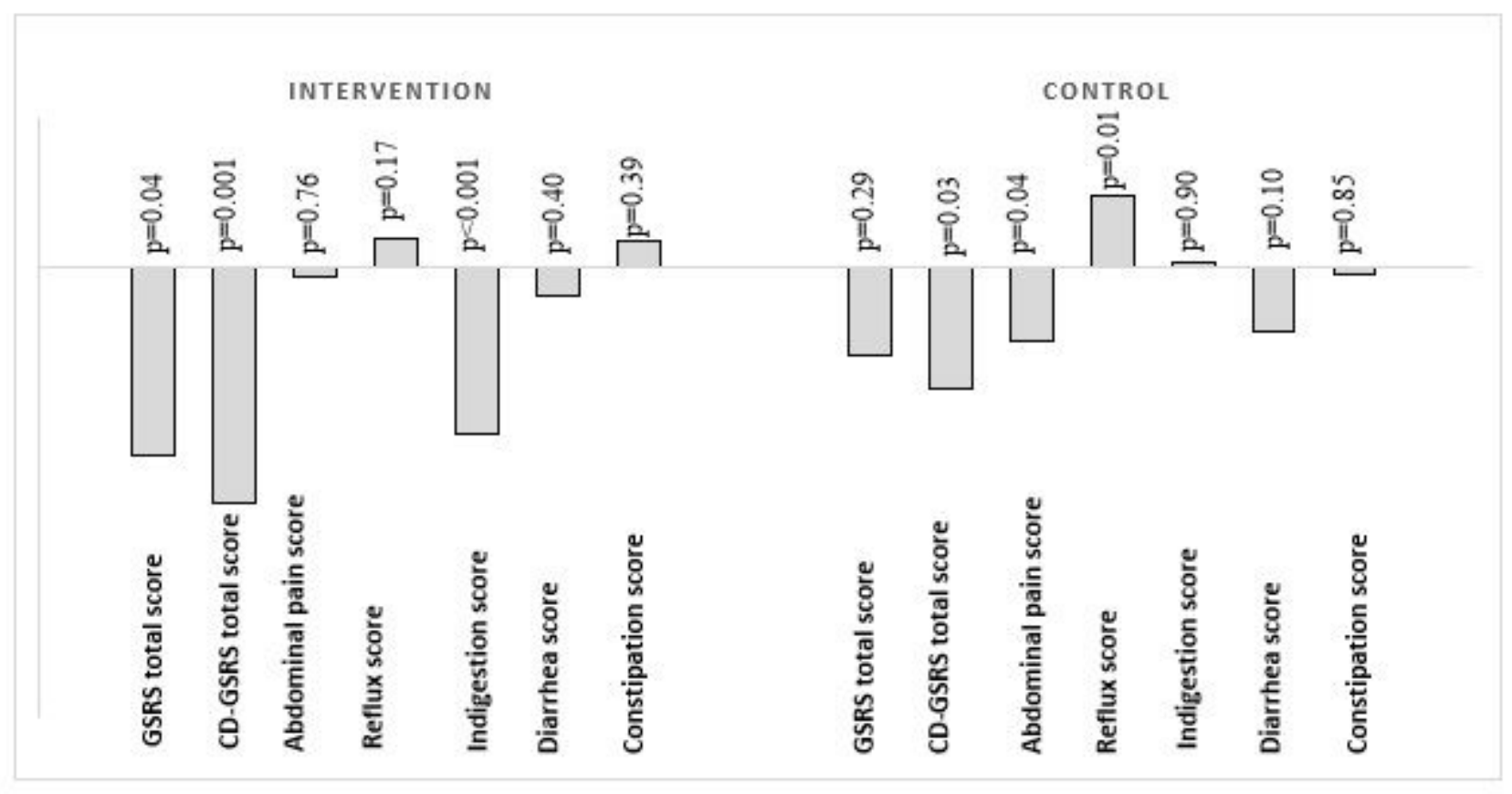

Figure 2

Mean changes from baseline of GSRS scores and subscores stratified by groups CD: celiac disease * $p$ value of ANCOVA test for comparing post-intervention values with adjusting for age, sex, disease duration, education level, and baseline value.

\section{Supplementary Files}

This is a list of supplementary files associated with this preprint. Click to download.

- CONSORT2010Checklist2.doc 